Rapport - Société canadienne d'histoire de l'Église catholique

\title{
Un Jésuite ambassadeur
}

\section{Honorius Provost}

Volume 32, 1965

URI : https://id.erudit.org/iderudit/1007337ar

DOI : https://doi.org/10.7202/1007337ar

Aller au sommaire du numéro

Éditeur(s)

Les Éditions Historia Ecclesiæ Catholicæ Canadensis Inc.

ISSN

0318-6148 (imprimé)

1927-7075 (numérique)

Découvrir la revue

Citer cet article

Provost, H. (1965). Un Jésuite ambassadeur. Rapport - Société canadienne

d'histoire de l'Église catholique, 32, 101-112. https://doi.org/10.7202/1007337ar

Tous droits réservés ( Inc., 1966
Ce document est protégé par la loi sur le droit d'auteur. L’utilisation des services d'Érudit (y compris la reproduction) est assujettie à sa politique d'utilisation que vous pouvez consulter en ligne.

https://apropos.erudit.org/fr/usagers/politique-dutilisation/ 


\section{Un Jésuite ambassadeur}

\section{SOMMAIRE}

Insistance des Abénaquis pour un missionnaire - Le Père Druillettes les accompagne en 1646 - Mission de l'Assomption - Mission suspendue - Première ambassade en 1650 - Seconde ambassade en 1651 - Mission et négociations sont abandonnées

Quelles qu'aient été, en 1629, les visées de Champlain, en envoyant un messager auprès des Abénaquis, cet échange de promesses et de civilités fut tragiquement interrompu par la reddition de Québec entre les mains des frères Kirk cette année-là et le départ du chef de la petite colonie.

Il faut filer jusqu'à 1637, sous M. de Montmagny, pour entendre parler de nouveau de ces sauvages. Mais, alors, les Français n'ont plus besoin d'eux et eux-mêmes n'ont plus besoin des Français, car, ils ont dans leur pays le voisinage des Anglais qui les satisfait. Leur venue à Québec est même considérée comme importune et l'on se figure qu'ils viennent rafler les fourrures des autres tribus pour les revendre aux Anglais à meilleur compte. Ils apportent, en effet, de la porcelaine obtenue sur une autre voie commerciale. Les Relations des Jésuites nous disent qu'en 1637, ils sont remontés jusqu'aux Trois-Rivières, pour rencontrer les Algonquins, malgré la défense expresse du Gouverneur. - Comme nos Sauvages vont parfois au païs des Abenaquois, ceux-ci les veulent aussi venir visiter à Kébec et plus haut. Mais cé n'est pas le bien de Messieurs les (Cent) Associez, car ces barbares viennent enlever les castors de ces contrées pour les porter ailleurs ${ }^{1}$. Cependant, on fit fouiller leurs cabanes, aux Trois-Rivières, et on ne trouva d'autre objet de contrebande que trois arquebuses, qui leur furent enlevées. Si M. de Montmagny avait écouté les Montagnais, ceux-ci auraient levé les armes contre ces étrangers et leur auraient fermé la voie du retour en leur pays. On était donc loin, pour alors, des bonnes relations entamées par Champlain.

Les conditions paraissent avoir été les mêmes en 1640, quand arriva inopinément, par la Chaudière, un aventurier anglais, le capitaine Young. En 1641, c'est une autre esclandre qui révèle toujours le même état d'esprit. Un algonquin polygame de la mission de Sillery s'en était allé cacher sa débauche chez les Abénaquis et y avait été tué, durant l'hiver, dans une rixe avec un sauvage enivré. Au

1 Relations des Jésuites, Québec, 1858, Relation de 1637, p. 86. 
printemps, deux abénaquis, envoyés par leur nation, vinrent aux TroisRivières pour présenter excuses et réparation aux parents de la victime. Mais, à leur arrivée, ils eussent été sûrement massacrés, sans la protection de quelques militaires de Québec et des sauvages chrétiens de Sillery, qui les avaient escortés.

Cependant les dispositions des Abénaquis eux-mêmes n'étaient pas mauvaises et leurs mœurs relativement douces les inclinaient vers l'alliance des Français. Ce qui les frappait surtout, c'était la transformation profonde onérée nar la re religion dana le caractère et le comp̣ortement des sauvages chrétiens groupés dans la réduction de Sillery. Ils les enviaient secrètement, eux qui, n'ayant d'autre voisinage que celui des trafiquants anglais dépourvus de prosélytisme, en recevaient plus d'occasions de se mal conduire que d'exhortations à mieux vivre.

Ces dispositions allaient bientôt faire pencher la balance et les rallier au parti des Français, quoique sans animosité ni rupture immédiate avec les Anglais, car les deux colonies voisines vivaient alors en bons termes. Ce qui prouve bien que la Nouvelle-Angleterre, en négligeant au début le sort de cette tribu, qu'elle avait dans sa main, a été la première cause de son retournement contre elle et de tous les malheurs que la guerre indienne devait faire pleuvoir sur la contrée pendant près d'un siècle.

Chose curieuse autant qu'édifiante, le précurseur de la bonne nouvelle aux Abénaquis ne fut ni un missionnaire ni un français, mais un sauvage chrétien nommé Charles Meïaschawat, chef montagnais converti en 1641. Il se vit d'abord confier le soin de reconduire dans leur région, en 1642, un prisonnier racheté des Algonquins, et il en profita pour hiverner chez les Abénaquis et leur prêcher l'Evangile. Un de leurs chefs le suivit jusqu'à Sillery, au printemps de 1643, pour s'y préparer au baptême ${ }^{2}$. Non content de ce premier succès, le bon Charles répéta son geste en 1644. "Il a pris la résolution d'y retourner cette année, nous dit la Relation, non pour autre fin que pour leur prescher Jésus-Christ; il nous vient communiquer son dessein. Il n'y a point de Père chez les Abnaquiois, nous dit-il, personne ne les enseigne, vous autres n'y pouvez pas aller, j'ay pitié de ces pauvres gens qui se damnent : je m'en vais les voir, je leur apprendray ce que vous m'avez appris... Je n'ay point d'esprit, mais si Dieu se veut servir de moy, il m'en donnera et nous ferons tous deux des merveilles. »

Les fruits ne se firent pas attendre : d'autres abénaquis vinrent se faire baptiser et séjourner à la mission Saint-Joseph de Sillery, rivalisant de ferveur avec les autres néophytes. Et ils se firent à leur tour des apôtres auprès de leurs compatriotes. «Ces nouveaux chrestiens, lit-on dans la Relation de 1646, prirent résolution ce printemps dernier de faire une course en leur païs, d'y publier la Foy, et de scavoir des principaux de leur nation, s'ils n'auroient point pour agréable de pres-

2 Ibid., Relation de 1643, p. 5 et 20. 
ter l'oreille aux Prédicateurs de l'Evangile ${ }^{3}$, Le 4 juillet, " deux eapitaines Abnaquiois, dont le principal qui estoit chrestien se nommait Claude... vinrent trouver M. le Gouverneur pour le prier de faire en sorte qu'une robe noire allast aux Abnaquiois pour les instruire; que cela estant une fois, ils ne viendroient plus icy \& ne donneroient aucun ombrage à $\mathrm{M}$. le Gouverneur pour la traite ${ }^{4}$. $\mathrm{Le}$ Supérieur des Jésuites remit la décision sur ce sujet à l'automne. Mais, les autres chrétiens partis le printemps revinrent avec des compatriotes encore payens, le jour de l'Assomption, et firent de telles instances, au nom de toute la nation, qu'ils gagnèrent leur point. La fondation d'une mission dans leur pays fut décidée, sous le vocable de l'Assomption.

Assez curieusement, on demeurait sceptique sur le succès de cette fondation. Dans la Relation, le narrateur écrit : "Le Pere Gabriel Dreuillettes, qui à desja vescu permy les Algonquins dans leurs grandes courses est allé passer le plus fascheux temps de l'année avec ces Abnaquiois, bien résolu de vivre et de mourir en la croix de Jésus-Christ. Il pourra pleinement satisfaire aux désirs qu'il a de souffrir, c'est ce qu'il peut attendre de plus constant et de plus asseuré parmy ces peuples ${ }^{5}$. ${ }^{*}$ Et le Journal des Jésuites (p. 64) est-il assez laconique, en notant, le 29 août : a Partit seul de françois pour la mission de l'Assomption aux Abnaquiois, le P. Gabriel Drüilletes, avec deux canots de sauvages dont le chef estoit Claude, bon chrestien ${ }^{6}$. .

$\mathrm{Au}$ reste, les voyages du Père Druillettes en Nouvelle-Angleterre sont bien connus depuis longtemps. Les historiens canadiens et américains les ont étudiés à fond ? Tous les documents originaux sur la question ont été publiés 8 .

Cependant, pour l'intérêt qu'elle présente, nous donnons ci-après une petite pièce moins connue, parce qu'elle a été depuis peu exhumée des archives. Elle s'intitule : "Ordre et certificat pour le R.P. drouillet de la Compagnie de Jésus, 1646 - Charles Huault de Monmagny Chev de l'ordre Sainct Jean de Hierusalem Gouverneur et Lieutenant general pour le Roy en la Nouvelle france, Certiffions a tous qu'il appartiendra que le Reverend père Gabriel Drouillet Religieu de la Comp ${ }^{\text {n10 }}$ de Jesus est party de Quebeq, de nostre consentm ${ }^{t}$, pour aller au pays des sauvages abénaquiois et autres lieux circonvoisins, pour tascher de donner

3 P. 18.

4 Journal des Jésuites, édition Laverdière et Casgrain, Québec, 1871, p. 57.

- Relation de 1646, p. 19.

- Faut-il écrire Dreuillettes ou Druillettes? Jacques Viger assure que le Père signait de la seconde façon.

7 La meilleure étude, à notre avis, se trouve dans l'ouvrage de Sœur Mary Celeste Leger, The Catholic Indian Missions in Maine (1611-1820), Washington, D.C., 1929, X-184 p.

8 On les trouvera surtout dans Twharres, Jesuit Relations and Allied Documents, 73 vols., Cleveland, 1896-1901; aussi dans les Documents relatifs à la Nouvelle.France, 4 vols., Québec, 1883, et dans Hazard Historical Collections..., 2 vols., Philadelphie, 1729-1794. 
aux sauvages la cognoissance du vray dieu, selon le desir. \& volonté de Sa Majesté tres Chrestienne et suivant l'institut de son ordre. S'y prions tous gouverneurs et capitaines des places par ou il pourra passer de luy prester toute assistance et confort, le laisser passer et repasser à sa volonté - Faict au fort Sainct Louis de Quebeq le vingt huict Aoust mil six cent quatante six.

\section{Par commandement de Monsieur le Gouverneur ${ }^{9}$."}

Cette sorte de passe-port donné par le Gouverneur au Père Druil.

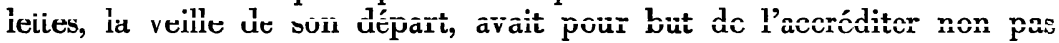
auprès des sauvages, mais auprès des Anglais, qu'on savait' tenir la direction de cette contrée. C'était donc un commencement de relations diplomatiques, dont, jusque là, on ne trouvait aucune mention.

Les péripéties du voyage ainsi entrepris en 1646 ne nous sont pas bien connues. L'auteur des Relations esquive le sujet' : "Je ne dis rien des difficultés qu'il faut essuyer dans un voyage de neuf à dix mois, où on rencontre des rivières serrées de rochers, où les vaisseaux qui vous portent ne sont que d'écorce $(\ldots)$ où il faut porter sa maison, son vivre et son couvert $(\ldots)$ où on diroit que tous les chemins conduisent en Enfer, tant ils sont affreux, et cependant ils menent en Paradis ceux qui ayment les Croix dont ils sont parsemez ${ }^{10}$. "Comme on le voit, ces écrits de propagande tournent aisément au lyrisme.

On connaît tout de même les allées et venues du missionnaire et ses oeuvres de zèle, durant ce premier séjour aux Abénaquis. Les historiens conviennent ${ }^{11}$ qu'il remonta d'abord la rivière Chaudière, le chemin traditionnel. Puis il descendit la Dead River et le Kennebec, prit contact avec le premier village sauvage de Narantsouack, continua sa route, guidé par son interprète indien, passa quelque temps au poste anglais de Kinibeki (Augusta), ou Coussinoc, puis descendit jusqu'à la mer, où il visita amicalement sept ou huit autres établissements. Ainsi rendu à proximité de l'Acadie, le Père se fit conduire au premier poste français, Pentagoët, à l'embouchure de la rivière Penobscot. Il y fut reçu à bras ouverts par les Pères Capucins, missionnaires attitrés de l'Ácadie, et par le Sieur de Chaste, commandant du poste, qui le combla de politesses et de provisions. Comme on était en bonnes relations avec la Nouvelle-Angleterre, il lui donna même des lettres de recommandation très louangeuses, pour le commandant du poste de traite de Coussinoc.

Le Père revint ensuite sur ses pas. Au retour, il « remonta une lieüe plus haut que Kinibeki, où les Sauvages se rassemblèrent au nombre de quinze grandes cabanes; ils luy bastirent une petite Chapelle de planches faite à leur mode ${ }^{12}$. Père les accompagna pour la chasse sur le haut de la rivière, au grand

9 Archives du Séminaire de Québec, documents Faribault, $\mathrm{n}^{\circ} 160$.

10 Relation de 1647, p. 51.

11 Ferland, Histoire du Canada, 2 vols., Québec, 1861, vol. I, p. 347.

12 Relation de 1647, p. 52. 
lac dénommé aujourd'hui le lac Moosehead. Telle fut la première mission de l'Assomption, où le Père Druillettes passa l'hiver à évangéliser avec grand succès. Il en revint le 16 juin 1647, d'après le Journal des Jésuites, toujours aussi avare de ses mots : "Arriva le P. Druilletes à Sillery de son voyage après plus de 9 moys d'hyvernement avec les sauvages Abnaquiois; ce qui en peut êstre su se trouvera dans les Archives ${ }^{13}$. D Malheureusement pour l'histoire, les archives des Jésuites sont en majeure partie disparues. Avec le secours des Relations et du Journal, on a pu cependant conserver le souvenir de cette aventure.

Les succès du Père Druillettes auraient dû provoquer son retour immédiat aux Abénaquis et les sauvages eux-mêmes ne manquèrent pas de le redemander avec instance. Mais le Supérieur des Jésuites, sur les entrefaites, reçut une lettre des Capucins de Pentagoët, apportée à Québec, les priant de ne pas retourner dans leur juridiction de l'Acadie et, pour éviter toute friction, on décida d'obtempérer à cette demande ${ }^{14}$.

Privés de missionnaire résident, les Abénaquis se retournèrent davantage vers Québec, où leur présence, pourtant, continuait d'être redoutée et détestée. Les 16 et 17 juillet 1649 , ils arrivaient une trentaine. On leur signifia de ne plus revenir, sinon leurs marchandises seraient confisquées. Ils repartirent tout de même avec vingt paquets de castor; autant de perdu pour les marchands du Canada. Ils avaient apporté des lettres des Anglais, preuve que les correspondances diplomatiques étaient entretenues par cette voie ${ }^{15}$.

C'est le gouverneur de Boston, John Winthrop, dont la juridiction s'étendait partiellement sur la région de Kennebec, qui avait répondu à la première mission, semi-officielle, de 1646. Dès 1647, profitant sans doute du retour du Père Druillettes de sa mission, il avait écrit au gouverneur de la Nouvelle-France à Québec ${ }^{16}$. Winthrop était mort en février ou mars 1649; mais son fils, un des principaux magistrats du Connecticut, nourrissait les mêmes dispositions favorables. Les Capucins de Pentagoët eux-mêmes, sous la direction d'un nouveau supérieur, le Père Cosme de Mante, entendirent les abjurgations des Abénaquis et, revenant sur leur prétention, remirent aux Jésuites la liberté d'action sur la rivière Kennebec ${ }^{17}$.

Tant et si bien que le Père Druillettes put repartir pour sa mission de l'Assomption, le premier septembre 1650. Cette fois, il n'était pas seul de français, puisque Jean Guérin, un «donné » des Jésuites l'accompagnait ${ }^{18}$. Il avait aussi avec lui le chef de la bourgade chrétienne de Sillery, Noël Negabamat. L'équipe constituait une véritable ambassade. On avait des passeports du gouverneur d'Ailleboust, des lettres

13 P. 88.

14 Journal des Jésuites, p. 91.

15 Ibid., p. 128.

16 Epistola Rev. P. Gabrielis Dreuillettes... ad Dominum Illustrissimum

Dominum Joannem Wintrop Scutarium, édité par Shea, New-York, 1869, p. 6.

17 Relation des Jésuites, année 1651, p. 14.

18 Journal des Jésuites (1650). 
de créance pour les gouverneurs et magistrats de toute la Nouvelle-Angleterre.

Comme pour le premier voyage, nous n'avons aucun détail sur l'allure et les incidents du trajet. Tout paraît s'être passé normalement. Le missionnaire, dans ce domaine de l'apostolat, en avait vu bien d'autres en fait de fatigues et d'incommodités. Parti le premier septembre 1650 , il arrivait " la veille Sainct Michel à cette habitaôon d'Anglois la plus haulte laquelle tant des Anglois et Sauvages est appelée Coussinoc ${ }^{19}$ \%. Ce poste (anjourd'hui Angusta) était situé. selon le Père, à seïze lieues de l'embouchure du Kennebec. Quinze ou seïze lieues en amont, il avait visité le village de Narantsouak et avait dû s'y arrêter quelque peu chez ses catéchumènes abénaquis.

Comme question de fait, plusieurs d'entre eux l'accompagnaient à Coussinoc, pour appuyer les premières démarches de l'ambassade. Le poste de traite, établi là dequis quelques années, appartenait à la colonie de Plymouth, en vertu d'une concession spéciale, et le commis du poste était John Winslow. Le Père Druillettes avait déjà gagné sa sympathie, lors du premier voyage. Appuyé par les sauvages, il obtint de lui une collaboration vraiment généreuse, celle de l'accompagner malgré son âge dans la poursuite de son voyage, afin de l'introduire aux gouverneurs du Massachusetts et de Plymouth. La précaution, d'ailleurs, n'était pas superflue, comme mesure de sauvegarde personnelle. Car, à Boston, la capitale du puritanisme, on venait de passer le "Massachusetts Âct against the Jesuits", qui aurait pu valoir au Père l'arrestation à vue, sinon la potence, n'eût-il pas été accompagné d'un envoyé officiel ou de quelque personnage reconnu comme responsable.

Le missionnaire se sépara alors de ses premiers compagnons et logea au poste, en attendant que John Winslow fût préparé à l'accompagner vers Boston. Ce n'était pas alors une mince affaire, et, pour plus de misère, les voyageurs, sans embarcation à leur disposition, durent faire par terre les dix premières lieues du trajet. Cela donna toutefois au Père Druillettes l'occasion d'apprécier encore mieux le dévouement de son mentor. Il pourra dire ensuite qu'il a trouvé en lui plus de générosité qu'il eût pu en rencontrer chez le meilleur compagnon français, pour faire réussir son voyage.

Le 25 novembre, les deux hommes pouvaient enfin prendre la mer, au fond de la baie de Merrymeeting ${ }^{20}$. Retardés par des vents contraires, ils n'arrivèrent que le 5 décembre à l'entrée de la baie de Massachussetts. Ils mirent pied à terre à Charleston et parvinrent enfin, le $8 \mathrm{du}$ mois, à Boston, où leur venue était signalée. C'est lo commandant militaire du lieu, le major-général Gibbons qui reçut le missionnaire, sur recommandation de Winslow, et qui lui offrit l'hos-

19 Narré du voyage faict pour la mission des Abnaquiois... ès années 1650. 1651. Par le R. Père Gabriel Dreuillette de la Compagnie de Jésus, édité par Shea, 33 p., New-York, 1855, p. 2.

20 Cette baie est un élargissement et comme l'estuaire de la rivière Kennebec. 
pitalité de sa maison, pour tout le temps de son séjour; il lui donna même la clef d'une chambre où il seroit tranquille pour dire la messe. Le lendemain, Gibbons accompagna le Père Druillettes chez le gouverneur du Massachusetts, nommé Dudley, résidant à une demi-lieue de Boston. L'entrevue revêtit une allure officielle : "Je luy présentai, écrit le Père, la lettre de créance de la part de M. le Gouverneur de Kébec et lui demandai audiance en qualité d'embassadeur et agent non seulement de $\mathrm{Mr}$ le gouverneur comme sa lettre portait, mais aussi de la part des catéchumènes abnaquois qui sont sous la juridiction de Pleymoutch qui est une autre colonie. C'estoit ce que mon pauvre habit et équipage témoignoit s'approchant plus d'un sauvage que d'un françois de médiocre condition ${ }^{21}$."

Le treize décembre, autre cérémonie. Le gouverneur avait convoqué spécialement les magistrats de la colonie et le représentant du peuple. Après avoir reçu le Père à dîner, ils entendirent l'exposé du but de l'ambassade, traduit par un interprète, en conférèrent entre eux et lui firent leur rapport au souper. Ces messieurs avaient déjà les usages de nos modernes politiciens!

Le Père Druillettes, toujours accompagné de Winslow, se rendit par mer le 22 décembre à Plymouth, rencontrer le gouverneur John Brentford, du New Hampshire. C'est, en effet, de cette colonie que relevait le poste de traite des Abénaquis sur le Kennebec et les action. naires du poste étaient en même temps les premiers magistrats de la colonie. Il fut plus facile au Père de faire écouter là sa requête, car les gens étaient directement intéressés. Il n'y demeura que deux jours et revint par terre à Boston, où il était de retour chez son hôte Gibbons, le 29 décembre. Il écrivit alors quelques lettres, en rapport avec sa mission, une particulièrement à son supérieur, le Père Lejeune, qu'il savait être pour lors en France. Il confia sa lettre à un navire anglais en partance de Boston pour Londres et cette lettre parvint à destination, puisque nous la possédons encore. Enfin, le 5 janvier, il prenait congé de Gibbons, qui le reconduisit jusqu'au port et le "recommanda fort particulièrement à Thomas Yau maistre d'un barcq qui partoit pour Kenebec ${ }^{22}$. En chemin, il fit un arrêt, se rendit à Salem visiter le fameux pasteur Endicott, qui le reçut avec les plus grands égards et lui parut très favorable à son ambassade. D'autres escales ou rencontres, et l'on fut de retour à la rivière Kennebec, le 8 février 1651. Le Père Druillettes reprenait là sa mission interrompue.

Son mandat d'ambassadeur avait comporté deux objectifs. Le premier, servant plus ou moins de prétexte au second, était de nouer des accords commerciaux avec les gens de Boston; l'autre était d'obtenir une alliance militaire contre les Iroquois, pour protéger les Indiens de la Nouvelle-Angleterre menacés de destruction. Toutes puissantes alors,

21 Lettre du père Druillettes à son supérieur, le père Lejeune, alors en France, le $1^{\text {er }}$ janvier 1651; copie aux archives du Séminaire de Québec, doc. Faribault, $\mathbf{n}^{\circ} 88$ : publiée dans Le Canada français, vol. 20, p. 941 à 949.

22 Narré du voyage..., p. 14. 
les Cinq Nations, qui avaient détruit les Hurons (1649), s'attaquaient aux autres alliés de la Nouvelle-France, que celle-ci, tremblant pour son propre sort, était incapable de protéger efficacement! Quelle réponse le Père en rapportait-il ? Aucun engagement formel, car les gouvernements des quatre colonies confédérées de la Nouvelle-Angleterre (Massachusetts. New Hampshire, Connecticut et Rhode-Island) ne devaient se réunir qu'en septembre suivant. Mais tous les individus consultés avaient paru favorables et s'étaient laissé impressionner, apparemment, nar les plaidoyers de l'ambassadeur. John Winslow, arrivant à son tour à Coussinoc, le 13 avril, assura que l'opinion, du côté de Plymouth et de Boston continuait à s'orienter dans le même sens. Du côté des sauvages, un enquêteur délégué par le Père, avec des présents, à l'automne précédent, revenait, le 24 avril, avec l'alliance assurée des principales tribus de la Nouvelle-Angleterre. Le succès de la mission ne paraissait plus douteux.

A Québec on en savait déjà quelque chose. En laissant le Père à Coussinoc, l'automne précédent, Noël Negabamat était rentré à Sillery, dès le 18 octobre, rapportant des lettres et des nouvelles. Revenu à sa mission, après le voyage de Boston, le Père Druillettes dépêcha à Québec d'autres messagers, avec des documents relatifs à son ambassade. Enfin, ce fut le Père lui-même qui reparut, autour du 4 juin, ayant prolongé sa mission auprès des Abénaquis jusqu'à la première baisse des eaux ${ }^{23}$.

Les espérances conçues et les fruits promis par les premières démarches engagaient tout le monde, et le Père Druillettes le premier, à retourner le plus tôt possible. Les Abénaquis venus avec lui refusaient de repartir sans lui et ne lui allouaient que le minimum de séjour pour retremper ses forces spirituelles et physiques.

Concernant ce troisième voyage du jésuite ambassadeur, outre les données du Journal et des Relations des Jésuites, trois documents offciels ont été conservés ${ }^{24}$. Le Conseil de Québec, chargé alors des intérêts de la Nouvelle-France, avait vraiment à coeur de continuer les négociations si bien entamées avec les colonies du Sud. Il s'assembla spécialement à cet effet le 20 juin 1651, à 9 heures du matin. On vota une résolution pour déléguer un des conseillers du nom de Jean Godefroy en ambassade avec le Père Druillettes et l'on écrivit une lettre aux Commissaires de la Nouvelle-Angleterre touchant les deux objectifs déjà exposés; enfin on pria le gouverneur d'Ailleboust d'accorder luimême aux deux délégués une commission en conséquence. Ce sont les trois documents officiels conservés.

23 Journal des Jésuites, p. 144 et 154.

24 Texte français cité dans Charlevolx, Histoire générale de la NouvelleFrance, livre VII, et reproduit tant bien que mal dans Collections of the Maine Historical Society, Documentory history, second series, vol. IV, p. 433-436; version anglaise dans Documents Relative to the Colonial History of the State of New York, vol. IV, p. 5 et 6. 
Le départ s'effectua dès le 22 juin, de façon spectaculaire. Le Journal des Jésuites mentionne : « Le P. Druillettes, M. Godefroy et Jean Guerin partent avec les Abnaquinois et un Sokoquinois pour la nouvelle Angleterre, 7 ou 8 canots. Noel Tek8enimat ${ }^{25}$ est de la partie. C'était un beau départ, vraiment; mais la Providence avait des vues plus austères.

Pourquoi, en entreprenant ce troisième voyage, ne prit-on pas le même trajet que d'habitude? On avait pourtant assez de guides abénaquis. Pourquoi se fier, cette fois, à un guide etchemin de la rivière Saint-Jean ou de la rivière Penobscot? Peut-être la curiosité de voir du pays? Peut-être l'intention de rechercher un passage plus court ou plus avantageux? En tout cas, c'était de la prudence à courtes vues ou tout simplement de l'imprudence et on dut l'avouer par la suite : «Les Nautonniers et les Guides qui conduisaient le Père, écrit la Relation de l'année 1652, prirent des routes nouvelles qu'ils n'avoient jamais fréquentées, et nous avons sceu depuis, que tous ceux qui les avoient tenües, estoient ou morts de fatigue et de faim, ou avoient pensé mourir ».

Nous ne pouvons voir qu'il puisse être question d'une autre route que celle de la rivière Etchemin. Dans les eaux basses, elle n'est presque pas canotable. Le convoi perdit quinze jours dans des étapes extrêmement pénibles et, au moment où les provisions étaient complètement épuisées, on reconnut avec consternation qu'on s'était égaré : au lieu d'aller vers le pays des Abénaquis, on descendait vers l'est, sur la rivière Saint-Jean. Le père Druillettes fit halte pour célébrer la messe; il n'avait pas plus tôt fini, que Dieu manifesta visiblement sa protection en jetant à portée de fusil trois orignaux, que les voyageurs abattirent et avec lesquels ils refirent leurs provisions. Mais il fallut remonter la rivière Saint-Jean jusqu'à sa source, dans cette partie où elle ne porte guère les embarcations. Au long portage qu'il fallait faire ensuite, le guide etchemin voulait aller au plus court et descendre à Pentagoët par la Penobscot; mais ce n'était pas l'avis du convoi. On lui imposa d'aller avec les autres canots rejoindre les eaux du Kennebec; impatienté, il ne fit plus que maugréer et insulter le Père, comme s'il avait été la cause de ces désagréments; il l'obligea, pour alléger son canot, à se séparer de son domestique et à jeter même son petit bagage personnel. Le Père endurait avec patience. Enfin, après 23 ou 24 jours de pénibles fatigues, les Abénaquis de Narantsouak revoyaient leur "patriarche ", qu'ils croyaient perdu. On s'imagine leur réjouissance comme aussi leur indignation envers ce pauvre sauvage etchemin, tout confus, et qui aurait pu payer cher ses insolences, si le Père ne l'avait défendu auprès des néophytes, édifiés avec raison par ce bel exemple de charité chrétienne.

Le père Druillettes avait sûrement un tempérament robuste. A peine réconforté par l'hospitalité des Indiens, "il visita premièrement, comme note la Relation, les 12. ou 13. habitations ou bourgades de ces peuples, qui sont rangées en partie sur la rivière de Kennebek, que les

Autre nom de Noël Negabamat. 
François appellent vulgairement Quinibequi, et en partie sur la coste de l'Acadie, que les Anglois occupent; il fut partout reçeu comme un Ange descendu du Ciel ». On le priait de ne plus retourner à Québec avant dix ans, et le capitaine de Narantsouak, la principale bourgade, le fit accepter ou naturaliser comme un membre de la nation.

Mais le Père n'oubliait pas son ambassade et il se rendit sans trop tarder, accompagné de son associé Godefroy et de Noël Tekouerimat, jusqu'à Boston puis New-Haven, parce que tous les commissaires des colonies " angloises 》 confédérées se trouvaient alors rẻunis à ce dernier endroit. La démarche des deux envoyés a été notée de mémoire, quelques années après, vers 1660, par un certain Samuel Maverick, dans une espèce de description de la Nouvelle-Angleterre, conservée au British Museum, à Londres. Voici cette mention : "About 10 yeares since a Gentleman and a Fryer came down this way from Kebeck to us in New England to desire aide from us against the Mowake Indians who were and still are their deadly ennemies ${ }^{26}$. On lit, par ailleurs, dans la Relation de 1652, que « le Père fut à Boston, à Playmot, bref il parcourut quasi toute la nouvelle Angleterre, sans que les Anglois se missent beaucoup en peine de secourir ces pauvres nations qui leur sont voisines ». Ce fut une navrante déception de constater un revirement d'opinion inexplicable, chez des autorités qui paraissaient si sympathiques six mois auparavant. Les rapports de la délégation sont bien maigres. La réponse finale, semble-t-il, fut que les Colonies unies de la Nouvelle-Angleterre consentaient bien à négocier un traité de commerce libre avec la Nouvelle-France, ce qui leur eût été avantageux, en cas d'un conflit avec leur métropole; mais qu'ils n'avaient pas de propension, pas d'intérêt, pourrions-nous ajouter avec d'autres auteurs, à nouer une alliance offensive ou défensive contre les Iroquois ${ }^{27}$. C'est avec cette réponse que le père Druillettes s'en revint tristement vers ses bons néophytes abénaquis et, s'il n'eût écouté leurs supplications, il aurait filé droit vers Québec pour venir raconter lui-même sa déconvenue. Mais il se résolut à passer encore l'hiver dans sa mission et à laisser Noël et ses compagnons rentrer à domicile. Ils y arrivèrent le dernier jour d'août, « avec les lettres du Père Druillettes ${ }^{28}$ ».

Quel était le contenu de ces lettres? Encore une fois, nous ne le savons pas, car les archives tant des Jésuites que du Conseil de Québec sont disparues. Mais nous pouvons soupçonner qu'elles donnaient lieu à quelque appréhension puisque, trois jours après, suivant le Journal des Jésuites, "La Fleur de Paris, Tand8taiouk et un Abnaquinois algonquinizé partent pour aller quérir le P. Druillettes ". Cependant, il appert que le missionnaire se trouva quelque raison de séjourner plus longtemps chez les Abénaquis; il ne profita même pas de l'occasion

26 Massachusetts Historical Society, Proceedings, Second Series, vol. I, 1884-1885, p. $23 \mathrm{~s}$.

27 Sister Mary Celeste Leger. The Catholic Indian Missions in Maine, X-184, p., Washington, D.C., 1929, p. 47.

28 Journal des Jésuites. 
qu'il aurait eue de rentrer à Québec avec Godefroy, l'autre ambassadeur, lequel fut de retour le 30 octobre, apportant à son tour des lettres du missionnaire ${ }^{29}$.

* Prends donc courage, lui disaient les sauvages, demeure avec nous, puisque nous sommes prests de t'obeyr. Tu es nostre compatriote; nous sommes tous de mesme nation (...) Delivre-toi, et nous aussi, de la peine de tant de voyages, et si longs et si fascheux, qu'on ne scauroit rien porter avec soy; ce qui nous met souvent en danger de mourir de faim. Nous sommes tesmoins que les principaux Anglois de ces contrées te respectent. Les Patriarches de l'Acadie nous ont dit qu'ils t'avoient escrit, que tu pouvois revenir en nostre pays quand tu voudrois. Que deviendront ceux qui mourront sans Baptesme ou sans confession en ton absence ? Je vous advoue, dit le Père, qu'ils m'attendrirent, et si je n'eusse creu que Dieu me rappeloit à Kébec par la voix de mon Supérieur qui me mandait, les travaux les plus horribles ne m'auroient jamais arraché du pays de ceux que j'aime plus que moy-mesme ${ }^{30}$. .

Il se sépara cependant de ses chers néophytes, devenus presque tous chrétiens et se risqua à revenir à Québec en raquettes sur les neiges, au début du printemps. Pourquoi cette hâte et cette autre imprudence ? Peut-être qu'il avait encore accompagné les sauvages pour leur chasse d'hiver au lac Moosehead et qu'il trouva le trajet de la sorte notablement réduit. Mais il dut payer cher cette décision pour le retour car il faillit y laisser sa peau et ses os, tout ce qui lui restait, en somme, lorsqu'il déboucha enfin, le 30 mars 1652, " à la pointe de Lauzon avec Jean Guérin, après bien des fatigues ». Ces derniers mots sont la mention laconique faite au Journal des Jésuites. La Relation ajoute que « luy et tous ceux qui le ramenoient, pensèrent mourir de faim et de froid; quelques uns mesmes perdirent la vie dans les neiges et dans l'excez des fatigues qu'il faut assez souvent souffrir dedans ces courses. Le Père et son cher compagnon ont soustenu leur vie dix jours entiers sans rien manger, après avoir jeusné tout le Caresme. Enfin ils s'adviserent de faire bouillir leurs souliers, et ensuite la camisole du Père, qui estoit faite de cuir d'Elan, et les neiges se fondans, ils firent aussi bouillir les cordes ou les tresses des raquettes dont ils se servoient pour ne point enfoncer quand elles étoient hautes. Tout cela leur sembloit de bon goût : la grace donne un merveilleux assaisonnement aux amertumes prises pour Jésus-Christ. Bref, ils arrivèrent à Kebec le Lundy d'après Pasques, n'ayans ny force ny vigueur, qu'autant' que le zèle du salut des âmes en peut donner à un squelet ${ }^{31}$." Voilà, croyons-nous, du style qui ne manque pas de relief et de couleur; en effet, si le tableau n'a pas été noirci capricieusement, il y avait bien dans ce voyage le sujet d'une odyssée. Et ce devait être la fin de la première entreprise missionnaire sur le Kennebec, en même temps que des négociations avec la NouvelleAngleterre.

29 Ibid.

30 Relation de 1652 , p. 31.

31 Relation de 1652, p. 26. 
Nous ne saurions terminer cette étude en donnant une conclusion plus judicieuse et plus impartiale que celle d'un historien américain, devant la Société historique du Maine, le 15 mai $1879^{32}$, et nous traduisons ses paroles : "Les Indiens abénaquis, ou du moins ceux-là du Kennebec et vers l'ouest ont toujours été regardés comme de la juridiction des colonies anglaises, et jamais dans les limites de la NouvelleFrance. Les Anglais n'ont fait aucun effort pour les christianiser. Les travaux des Jésuites parmi eux furent le résultat (humainement parlant) non d'ıne entreprise roncertée, mais d'un hasard. Ils se tinrent éloignés de ce champ d'apostolat jusqu'à ce que l'appel au secours devînt si suppliant qu'on ne pût l'ignorer ou y résister. Leurs travaux n'eurent aucune teinte politique mais furent exécutés dans le plus pur esprit de charité religieuse. Les Indiens souhaitaient conserver l'alliance avec les Anglais et demandaient leur protection; mais on la leur refusa. A quoi s'attendre, alors, sinon à ce qui est arrivé dans la suite ? Les terribles conséquences de la guerre appelée la guerre française et indienne furent le résultat direct de l'aveuglement de nos ancêtres. Leurs desseins orgueilleux visaient à humilier la colonie croissante des Français, au nord, mais ils avaient laissé leurs frontières ouvertes de ce côté aux attaques d'un ennemi sauvage et irréconciliable.»

Abbé Honorius Provost, archiviste

Président de la Société historique de Québec

32 John Marshall Brown, The Mission of the Assumption, dans Collections of the Maine historical Society, Series II, Vol. I, p. 87-99. 